\title{
RESONANT TUNNELLING STUDIES OF CHAOS IN QUANTISED SYSTEMS
}

\author{
L. Eaves, T.M. Fromhold, P.B. Wilkinson and F.W. Sheard \\ Department of Physics, University of Nottingham, Nottingham NG7 2RD, UK
}

This paper gives a brief introductory overview of quantum chaology, with particular reference to recent experimental work involving the use of semiconductor heterostructures. In the presence of a tilted magnetic field, a double-barrier resonant-tunnelling device incorporating a quantum well produces a chaotic stadium for electron motion. The basic properties of this system are described. It is shown how resonant magnetotunnelling spectroscopy provides firm experimental evidence for the effect of scarred wave functions on a physically-measurable property, in this case the measured current-voltage characteristics of the device. The paper concludes with some speculations concerning for the development of this field.

PACS numbers: 72.20.My, 73.40.Gk, 85.30.Mn, 05.45.+b

\section{Quantum billiards, unstable orbits and scarred wave functions}

Quantum chaology has been defined by Berry [1] as the "study of semiclassical, but non-classical, behaviour of systems whose classical motion exhibits chaos". Much of the theoretical work in this field has focused on the classical motion and corresponding eigenstates of particles which are confined to move within a two-dimensional stadium (also commonly referred to as a billiard) [1-3]. Particles are assumed to bounce elastically off the walls of the stadium, with specular reflection. The motion within the confines of the stadium is normally assumed to be free and frictionless. Particles moving in square- and circular-shaped stadia have regular motion, with one or two characteristic periods; in mathematical terms, this means that the equation of motion is separable into two one-dimensional parts. Examples of stadia which give rise to chaotic motion are the Bunimovich stadium (a square area with two semicircular areas adjoined to opposite sides; for experimental stadia see Ref. [4]) and the Sinai billiard [5] (a square with a hard-walled circular "no-go" area in the centre). The properties of unstable but periodic classical orbits are of fundamental importance for the quantum chaology of these types of billiard $[1,2]$. These orbits are associated with regular clustering of the quantised energy levels of the system, which gives rise to energy-periodic fluctuations in the density of states. This effect is described in terms of the well-known 
Gutzwiller trace formula [2], in which the energy spacing between clusters of levels, $\Delta E=h / \tau_{\mathrm{p}}$, where $\tau_{\mathrm{p}}$ is the period of the unstable orbit. Periodic unstable classical orbits are also closely associated with regions of enhanced probability density for some of the eigenfunctions of the quantised system. This effect is commonly termed "wave function scarring", because the classical trajectory somewhat mysteriously leaves its mark or "scar" on the quantum state [6].

At the experimental level, quantum chaos has been studied by investigating the spectra of hydrogen and related atoms in high magnetic fields [7-14], and by studying the ionization of atoms in the presence of large microwave electric fields [15]. Theoretical work by Du and Delos [8] has shown that both level clustering and scarring can contribute to the intensity oscillations of the photoabsorption spectrum of the highly-excited states of atomic hydrogen at high fields, an effect first reported by Garton and Tomkins [7]. However, this theory says nothing about the contribution of the individual discrete or quasi-discrete states of the system. Further work on the distribution of scar strengths over eigenstates has emphasised that the observable photoabsorption peaks in the chaotic regime, near the photoionization threshold of hydrogen, cannot in general be related to individual eigenstates. As noted by Heller recently [16], these atomic spectroscopy measurements only provide indirect evidence for the existence of scarred states.

\section{Quantum chaos in semiconductor heterostructures}

Although scarring effects have been observed in the resonant response of classical electromagnetic microwave cavities [17], the lack of direct experimental evidence in quantum systems for the effect of individual scarred wave functions on directly observable phenomena was the motivation of our recent experiments on quantum chaos [18]. Our aim was to create a chaotic stadium for electrons in the quantum regime. Our stadium consists of a wide quantum well, formed from a GaAs/AlAs heterostructure grown by molecular beam epitaxy (MBE), see Fig. 1. The GaAs quantum well is confined between two AlAs tunnel barriers, sufficiently thin to allow the passage of a tunnel current. This allowed us to study the eigenstates of the quantum well by measuring the peak in the current-voltage curves, $I(V)$, a technique known as resonant tunnelling spectroscopy.

We exploit a remarkable property of MBE, namely that it can produce interfaces which are flat to within about one atomic layer. These effectively act as specular reflectors for incident electrons. Since the de Broglie wavelength of an electron in these structures is typically $10 \mathrm{~nm}$ or more, the barrier has a quality equivalent to a $\lambda / 30$ mirror or lens in optics. It is worth noting that astronomical telescopes are regarded as of outstanding quality if they have $\lambda / 10$ optics!

An electron bouncing freely between the barriers of a quantum well has periodic motion with a constant period in the absence of dissipative processes. The application of a magnetic field makes the electron motion more complicated, but if the field is applied perpendicular to the plane of the barriers, the motion is still periodic, with a cyclotron motion superimposed upon the bouncing motion from wall to wall. Even if the magnetic field is applied parallel to the barriers, the motion is still periodic: i.e., at high fields, when the cyclotron radius is less than the width of the well, the electron simply skips along the barrier wall; at lower 
fields, the electron still bounces periodically off both barriers, but with a curved trajectory.

However, the magnetic field can induce chaotic motion when it is tilted at an angle to the barriers [18-24]. In this case, the cyclotron motion becomes "scrambled up" with successive bounces off the barrier walls. It is easy to demonstrate the existence of completely chaotic motion in this situation by generating Poincaré sections. This involves plotting successive values of the in-plane velocity components, $v_{x}, v_{y}$, as points in 2D space for successive hits on one of the barrier walls $[18,21]$. At a sufficiently large angle of tilt, $\theta$, from the normal to the barriers, the Poincare sections have a "dusty" appearance due to a random distribution of points [20]. Our chaotic stadium is formed by the two flat tunnel barriers and by an effectively parabolic or gutter-shaped magnetic potential produced by the Lorentz force on the electrons. The axis of the gutter must, of course, be set at an angle $\theta \neq 0$ or $90^{\circ}$ to the two flat barrier walls to achieve chaotic conditions.

The Hamiltonian describing this system is straightforward, since the magnetic field is incorporated in the usual way by means of the magnetic vector potential $[18,20]$. The effective-mass approximation is used to describe the motion of the electron in the conduction band. Since we investigate electrons injected into the quantum well through the tunnel barrier at quite high energies (up to around $0.5 \mathrm{eV}$ ) it is necessary to take into account the non-parabolicity of the conduction band of the GaAs quantum well. The degree of non-parabolicity can be directly measured by independent experiments at zero magnetic field. These experiments measure the peaks in the current-voltage characteristics, $I(V)$, corresponding to resonant tunnelling into the discrete subbands of the quantum well. Comparison with the calculated energy separation of the subbands provides us with a measure of the non-parabolicity effect [18].

The combination of flat barriers and well-defined magnetic potential provides what is in many respects an ideal stadium for studying chaos, since elastic processes originating from imperfections in the device (e.g. impurities, interface roughness) are essentially absent. However, the system has one drawback. Since tunnelling electrons are injected into the quantum well at high energies, they can relax their energy to the lattice by the emission of longitudinal optic (LO) phonons [19]. GaAs is a relatively polar material, so that the emission time is less than a picosecond. According to the uncertainty principle, this leads to an energy level broadening of the quantum well states by about $5-6 \mathrm{meV}$. In our original experiments, we employed wide quantum wells of width 60 and $120 \mathrm{~nm}$ [19-21]. In this case, the mean level separation is less than the linewidth. Hence, individual levels are not properly resolved and the effects of the chaotic motion reveal themselves only as a weak modulation in the density of electron states. The mean separation between levels can be increased by working with a narrower quantum well, e.g. $20 \mathrm{~nm}$, for which the individual levels are well-resolved. However, it is necessary to work at much higher magnetic fields in this case, in order to maintain the conditions of classical chaos. For this reason, our experiments [18] to investigate the effects of scarring of individual and well-resolved eigenstates were carried out using the pulsed magnetic fields up to 50 tesla at the Institute of Solid State Physics, University of Tokyo, in collaboration with N. Miura and T. Takamasu. 
Before describing the principle results of our experiments, we note two other recent experiments on quantum chaology in semiconductor spectra. Both of these involve the measurements of the magnetoresistance of high mobility two-dimensional electron gas systems which are patterned by electron beam lithography (EBL). Marcus et al. [4] fabricated a Bunimovich stadium in which the walls were formed by a negatively-biased electron gate fabricated by EBL. The stadium incorporated two openings through which current passed and across which voltage was measured. The magnetoresistance of this structure showed a complicated aperiodic oscillatory structure. Weak periodic fluctuations were also observed and associated with periodic unstable orbits. Weiss and co-workers [25] investigated the magnetoresistance of an anti-dot superlattice, also formed by EBL. In this case, electrons chaotically scattered by the antidots carry the current through the device, whereas periodic states are bound to one or more antidots, and do not conduct [25, 26]. In both these experiments, the conductivity is measured in the linear regime, so that transport occurs very close to the equilibrium chemical potential of the degenerate 2DEG. Hence at low temperatures the mean free path of carriers is not significantly inhibited by phonon emission and absorption processes, as in the case of our resonant tunnelling device. On the other hand, the disadvantage of both systems is that unpredictable scattering processes can mask effects arising from chaos. Such scattering can arise through the presence of charged impurity scattering or, perhaps more importantly, due to the limitation of EBL in defining correctly-shaped stadia and antidot arrays; i.e. the stadium walls are not flat to within a de Broglie wavelength. The experiments on EBL-fabricated stadia and antidot arrays and our own work on tunnelling structures therefore complement one another in some respects.

\section{Experimental details}

The resonant-tunnelling diode (RTD) structure shown in Fig. 1a is grown by molecular beam epitaxy and processed into circular mesas with ohmic contacts to the top and bottom doped layers. The two $\left(\mathrm{Al}_{0.4} \mathrm{Ga}_{0.6}\right)$ As layers act as $330 \mathrm{meV}$ high potential barriers to the motion of electrons through the device. Under an applied bias voltage $V$, electrons tunnel from the left-hand (emitter) contact into the quantum well (QW) formed between the two $\left(\mathrm{Al}_{0.4} \mathrm{Ga}_{0.6}\right) \mathrm{As}$ barrier layers (Fig. 1b). The electrons in the emitter form a two-dimensional electron gas (2DEG), in which they are free to move in the plane of the barrier $(y-z)$, but confined within a narrow accumulation channel of width $\simeq 15 \mathrm{~nm}$ in the $x$-direction. This spatial confinement quantises the energy associated with motion along the $x$-direction. For the range of $V$ considered here, only the lowest energy level $\epsilon_{b}$ is populated. In our calculations, $\epsilon_{b}$ is found using a simple model for the potential variation through the device $[18,20]$.

When a magnetic field $B$ is applied at an angle $\theta$ to the $x$-direction (see inset) the energy of electrons in the 2DEG associated with motion in the $y-z$ plane is quantised into Landau levels. Only the lowest Landau level is populated and the total energy of the emitter bound state is $\epsilon_{2 \mathrm{DEG}}=\epsilon_{\mathrm{b}}+\hbar B e \cos \theta / 2 m^{*}$. Electrons in the QW are spatially confined by the two barriers and by the magnetic field. This confinement quantises the total energy of an electron in the QW into discrete 


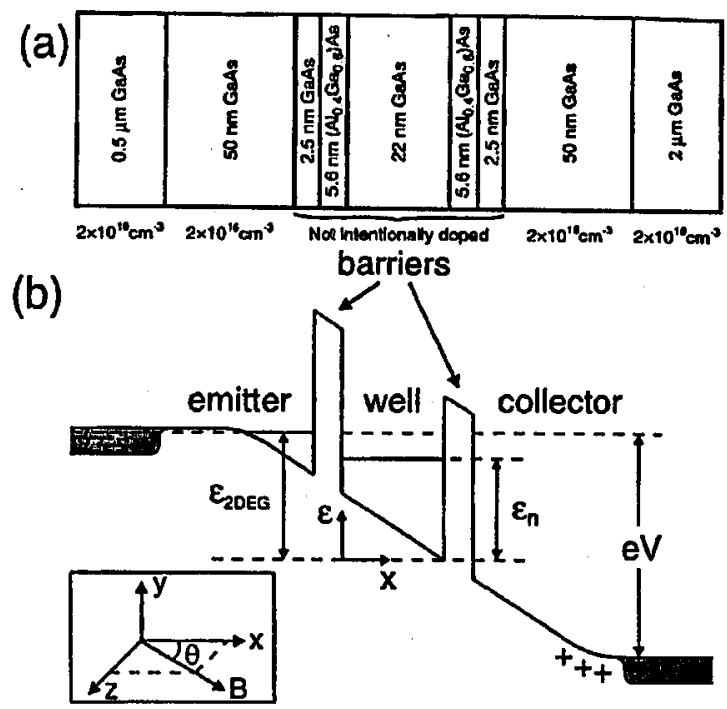

Fig. 1. (a) Composition of the resonant-tunnelling diode used in our experiments, showing layer thicknesses and concentrations of Si dopant. (b) Schematic variation of the potential energy of an electron at the conduction band edge with position $x$ normal to the layer interfaces. Inset: orientation of the tilted magnetic field.

energy levels $\epsilon_{n}(n=1,2,3 \ldots)$. When $V$ is increased, the emitter bound state scans the quantised energy level spectrum of the QW. Resonant tunnelling from the emitter into the $n$-th bound state of the QW occurs at the bias voltage for which $\epsilon_{2 \mathrm{DEG}}=\epsilon_{n}$.

Figure 2a shows the experimental $\mathrm{d} I / \mathrm{d} V$ versus $V$ characteristic for $\theta=40^{\circ}$ and $B=37 \mathrm{~T}$. A regular series of conductance peaks is observed with average voltage spacing $\Delta V \cong 87 \mathrm{mV}$. A derivative plot is used to emphasise the resonant peaks (arrowed). This is much greater than that expected from the mean level spacing in the $\mathrm{QW}$ which suggests that the tunnel current is dominated by a subset of levels. In the following section we consider the energy levels and eigenfunctions of the states in the quantum well and use them to understand the form of the current-voltage characteristics.

\section{Analysis of the data and discussion}

Under the conditions of our experiment, the quantised energy-level spectrum corresponds to the regime of predominantly strong classical chaos [18]. The level spectrum is shown in Fig. 3a. The density-of-states plot in Fig. 3b includes the level broadening expected for LO phonon emission. Details of the calculations are given elsewhere [20]. Close to the energy of the injected electrons $(\approx 280 \mathrm{meV})$, most individual energy levels produce well-resolved peaks in the density of levels plot $D(\epsilon)$. Periodic fluctuations in the density of levels can also be identified by locating the minima (arrowed) between broad peaks in $D(\epsilon)$. These are associated 


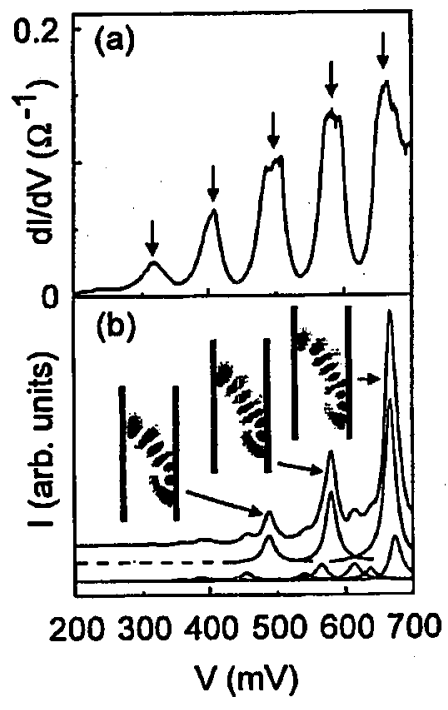

Fig. 2. (a) Experimental $\mathrm{d} I / \mathrm{d} V$ versus $V$ plot measured for a $100 \mu \mathrm{m}$ diameter mesa resonant tunnelling diode at $B=37 \mathrm{~T}$ and $\theta=40^{\circ}$. The principal resonances are approximately equally spaced in voltage, as indicated by the arrows. (b) Calculated $I(V)$ characteristic (top curve) and current contributions (middle and bottom curves) due to transitions into individual eigenstates of the $\mathrm{QW}$ when $\theta=40^{\circ}, B=37 \mathrm{~T}$. Strong periodic resonant peaks in $I(V)$ (arrowed) originate from the dominant current contributions (middle curves) produced by tunnelling transitions into individual scarred states with probability distributions in the $x-z$ plane shown inset. These scarred states are calculated at the peak of the current contributions and belong to the same sequence shown in Fig. 3c. The remaining current contributions (lower curves) generate only weak features in $I(V)$.

with unstable but periodic orbits in the QW via the Gutzwiller trace formula [2]. As $V$ is varied, resonant tunnelling into the energy levels $\epsilon_{n}$ of the QW might be expected to produce an irregular sequence of peaks in the current-voltage characteristic $I(V)$. Previous measurements on RTDs with $60 \mathrm{~nm}$ and $120 \mathrm{~nm}$ wide QWs in magnetic fields up to $11 \mathrm{~T}$, revealed quasiperiodic series of resonant peaks, which were attributed to Gutzwiller fluctuations in the density of levels associated with distinct unstable closed orbits in the QW $[19,21]$. In these studies, the QWs were too wide for individual energy levels to be resolved. However, our recent theoretical work [20] indicated that in certain regimes, the periodic tunnelling peaks observed for these structures were due to regularly spaced subsets of individual levels, whose eigenfunctions were scarred by particular closed orbits. In the present experiments, the QW is sufficiently narrow $(22 \mathrm{~nm}$ ) that individual levels can be resolved, although a much higher magnetic field is needed to ensure classically chaotic behaviour at $\theta=40^{\circ}$.

The current due to resonant tunnelling into the $n$-th state of the QW is determined by the transition rate $W_{n}$ from the occupied emitter states into the 


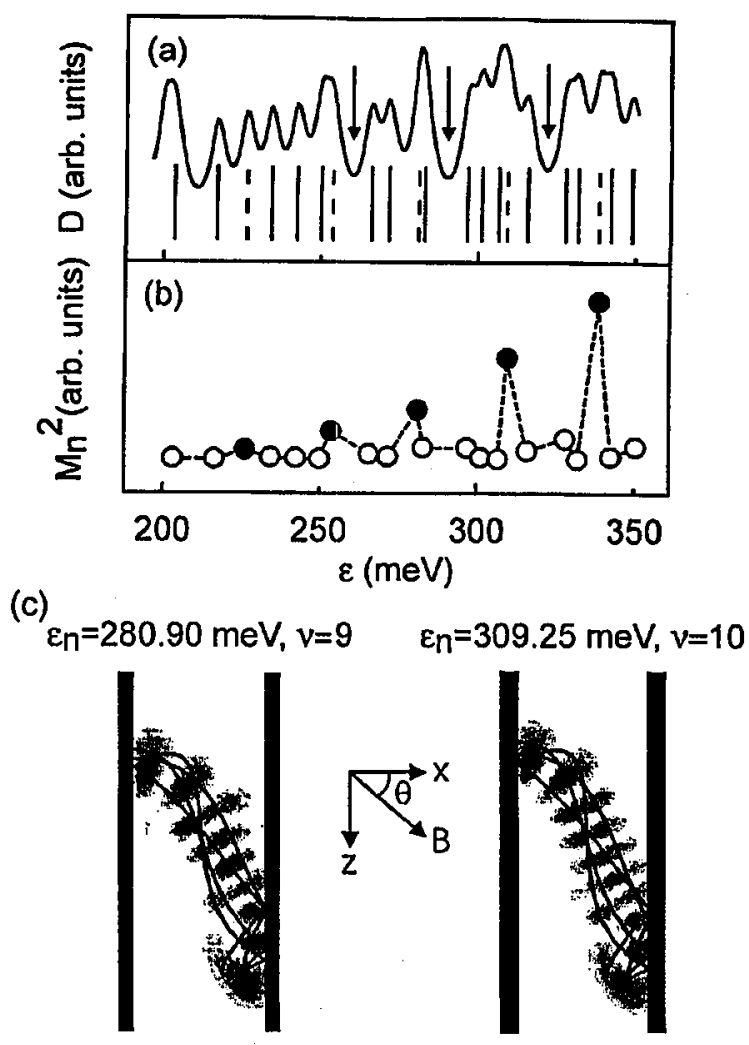

Fig. 3. (a) Vertical lines: quantised energy levels $\epsilon_{n}$ calculated for the $22 \mathrm{~nm}$ wide QW with $V=668 \mathrm{mV}, B=37 \mathrm{~T}, \theta=40^{\circ}$. Solid curve: density of levels $D(\epsilon)$ obtained by broadening each energy level to a width of $6 \mathrm{meV}$ consistent with the finite lifetime of about 0.1 ps imposed by the emission of longitudinal optic phonons. Arrows show successive minima in $D(\epsilon)$ associated with Gutzwiller fuctuations produced by unstable periodic orbits in the $\mathrm{QW}$ in which the electron makes two or three successive collisions on the $\mathrm{RH}$ barrier for each collision on the LH barrier. These orbits also produce strong scars in the wave functions corresponding to the subset of energy levels shown as vertical dashed lines. Orbits and scar patterns are shown in (c). (b) Open circles: squared matrix elements $M_{n}^{2}$ for tunnelling transitions into quantised energy levels shown in (a). Closed circles indicate matrix elements for transitions into a subset of individual scarred states, two of which are shown in (c). (c) Probability density plots (white background $=0$ ) of scarred QW eigenfunctions corresponding to energy levels and quantum numbers $\nu$ shown. Similar scar patterns are found in the wave functions corresponding to all the energy levels shown as dashed lines in (a). The thick black vertical lines indicate positions of barriers (thickness not to scale). Inset: orientation of magnetic field $B$ in the $x-z$ plane. In our chosen gauge given by the vector potential $A=(0, x B \sin \theta-$ $z B \cos \theta, 0$ ), the probability density depends only on the $x$ - and $z$-coordinates (axes inset). The trajectories of three distinct unstable periodic orbits which contribute to the scar patterns are shown projected on the $x-z$ plane as solid continuous curves. 
almost empty QW state [20]. In the Bardeen transfer-Hamiltonian description of resonant tunnelling, $W_{n} \propto M_{n}^{2}$, where the matrix element $M_{n}$ depends on the overlap between the wave functions of the emitter and QW states in the tunnel barrier. We have calculated the transition matrix elements using a simple, separable wave function for the emitter bound state. The variation of $M_{n}^{2}$ with energy $\epsilon_{n}$ is shown in Fig. 3b. The matrix elements for transitions into a subset of almost equally-spaced energy levels (vertical dashed lines in Fig. 3a) are much larger than for the remaining states. The reason for this can be seen by examining the probability distributions of the corresponding wave functions, two of which are shown in Fig. 3c. These wave functions reveal remarkably clear scars of three distinct closed orbits (overlaid) in which the electron makes either two or three successive collisions on the right hand (RH) barrier per period. The energies of these scarred states can be accurately located using a Bohr-Sommerfeld quantization of the classical action $S(\epsilon)=(\nu+\phi) h$ along the scarring orbits [20,27, 28] (the different orbits have almost the same classical action for given $\epsilon$ ), where $\phi \approx 1$, and the quantum number $\nu$ gives the number of antinodes in the scar pattern along the classical path [20]. The energy spacing $\Delta \epsilon_{\mathrm{P}} \approx 28.4 \mathrm{meV}$ of the scarred states and also of the Gutzwiller fluctuations in Fig. 3a, is very close to the expected semiclassical value of $h / T_{\mathrm{P}} \approx 28.9 \mathrm{meV}$, obtained from the period $T_{\mathrm{P}} \approx 0.143 \mathrm{ps}$ of the scarring orbits. The strong spatial overlap between the emitter state and the subset of scarred states is a direct consequence of the scarring, which localises the probability density of the QW state in the vicinity of the classical orbit. For unscarred states, the probability is more diffused and the overlap is much weaker. In order to illustrate the striking difference in the forms of scarred and unscarred wave functions, Fig. 4 shows an unscarred state at energy $\epsilon=315.5 \mathrm{meV}$ which is the wave function probability map of the next-higher energy level adjacent to the scarred state shown in Fig. 3c, right (309.25 meV). It has a strongly irregular antinode pattern and shows no indication of scarring. The tunnelling probability into this type of state is clearly much less than that into the scarred states, which have a strong single antinode adjacent to the emitter (LH) barrier.

We note that the scarring phenomenon is particularly strong for the quantum well in a tilted magnetic field due to special dynamical properties which are not shared by atoms. In particular, the scarring orbits have small Lyapunov exponents for a wide range of field strengths and orientation [29], and there are no spatially closed but aperiodic orbits which are known to complicate the scar patterns of hydrogenic atoms [12].

We now calculate the tunnelling characteristic from the transition rates studied above. Figure $4 \mathrm{~b}$ shows the theoretical $I(V)$ curve (top) together with the current contributions due to transitions into individual states in the QW (middle and lower). The dominant current contributions (middle curves) clearly originate from transitions into scarred states (shown in inset) and generate strong resonant peaks in $I(V)$ (arrowed). The predicted peak spacing $\Delta V \cong 94 \mathrm{mV}$ is in good agreement with the experimental results. This provides clear evidence that the observed resonant peaks originate from transitions into the subset of scarred states shown in Figs. 3 and $4 \mathrm{~b}$. Note that transitions into other QW states produce no significant visible resonant features. We stress that the large voltage spacing and periodic 


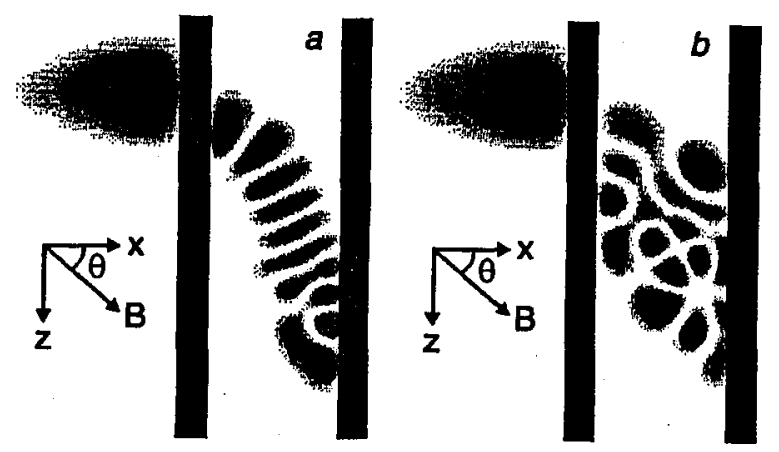

Fig. 4. Probability density plot of eigenstates for the $22 \mathrm{~nm}$-wide quantum well at energies (a) $\varepsilon_{n}=309.25 \mathrm{meV}$ and (b) $\varepsilon_{n+1}=315.5 \mathrm{meV}$. The probability map of the eigenstate shown in (a) is also shown in Fig. 3c and is scarred. The state shown in (b) reveals no trace of scarring. Dark indicates high probability density. The thick vertical lines represent the barriers, drawn to scale. The calculation is done at a bias voltage of $668 \mathrm{mV}$. The plots are drawn in the $x-z$ plane, where the $x$-axis is perpendicular to the confining barriers. A magnetic field of $37 \mathrm{~T}$ is applied in the $x-z$ plane at an angle of $40^{\circ}$ to the $x$-axis. The figure also shows the egg-shaped distribution functions of the lowest Landau level emitter states to the left of the left-hand barrier.

distribution of the observed resonant peaks exclude the possibility that they originate from transitions into adjacent energy levels in the QW. A quasi-selection rule based on the periodic scarring of individual wave functions is essential to account for the experimental data.

\section{Conclusions}

We have shown how the almost atomically-flat interfaces of MBE quantum well structures, combined with high magnetic fields, can be used to create a new type of stadium which allows us to directly probe the manifestations of the classically chaotic motion on the quantised states of the system. By measuring the resonant tunnelling current-voltage characteristics of the device, we are able to investigate the distribution of scarred eigenstates in a QW with strongly chaotic electron dynamics. Energy- and momentum-conserving tunnelling transitions into a subset of scarred states in the QW have been shown to generate dominant peaks in $I(V)$, which provide direct experimental evidence for the periodic scarring of individual wave functions in a nonintegrable quantum system [18]. To our knowledge, this is the only quantum system in which a periodic effect originating from individual scarred eigenstates is observed in experiment. The effect is clearly distinguishable from that due to Gutzwiller fluctuations in the density of energy levels.

We note that much recent theoretical work on the quantum mechanics of classically chaotic systems has focused on the universality of the energy level spectra (see, for example, Ref. [2]). This work reveals beautiful agreement between the energy level statistics of quantum chaotic systems and the predictions of random matrix theory. However, our experiments show clearly that physically-measurable 
properties such as the tunnel current flowing through a system depend not only on the form of the energy level spectrum, but also critically on the spatial form of the eigenfunctions. Perhaps theoreticians need to bear this point in mind when considering the universality of quantum chaotic systems.

Finally, we note that recent work has shown that the ideas of quantum chaos can be used to analyse the distribution of electric field intensity around fibre optic cables [30]. The strong analogy between quantum mechanical and optical interference effects, and classical chaotic orbits and light rays suggests that our understanding of quantum chaology could find applications in new optical technologies, such as fibre optic communications and photonics.

\section{Acknowledgment}

This work is supported by EPSRC. Two of us (L.E. and T.M.F.) are supported by EPSRC Senior and Advanced Fellowships, respectively. The experimental work described in this paper was carried out in collaboration with our colleagues at the Institute of Solid State Physics, University of Tokyo, Japan, Prof. N. Miura and Dr. T. Takamasu.

\section{References}

[1] M.V. Berry, Proc. R. Soc. Lond. A 413, 183 (1987).

[2] M.C. Gutzwiller, Chaos in Classical and Quantum Mechanics, Springer, New York 1990; L.E. Reichl, The Transition to Chaos, Springer, New York 1992.

[3] E.J. Heller, S. Tomsovic, Physics Today July, 38 (1993).

[4] C.M. Marcus, A.J. Rimberg, R.M. Westervelt, P.F. Hopkins, A.C. Gossard, Phys. Rev. Lett. 69, 506 (1992).

[5] Ya.G. Sinai, Russ. Math. Survey 25, 137 (1970).

[6] E.J. Heller, Phys. Rev. Lett. 53, 1515 (1984).

[7] W.R.S. Garton, F.S. Tomkins, Astrophys. J. 158, 839 (1969).

[8] M.L. Du; J.B. Delos, Phys. Rev. A 38, 1896, 1913 (1988).

[9] K. Müller, D. Wintgen, J. Phys. B, Mol. Opt. Phys. 27, 2693 (1994).

[10] A. Holle, J. Main, G. Wiesbusch, H. Rottke, K.H. Welge, Phys. Rev. Lett. 61, 161 (1988).

[11] J. Main, G. Wiesbusch, A. Holle, K.H. Welge, Phys. Rev. Lett. 57, 2789 (1986).

[12] D. Wintgen, A. Hönig, Phys. Rev. Lett. 63, 1467 (1989).

[13] D. Wintgen, H. Friedrich, Phys. Rev. A 36, 131 (1987).

[14] H. Hasegawa, M. Robnik, G. Wunner, Prog. Theor. Phys. Suppl. 98, 198 (1989).

[15] R.V. Jensen, M.M. Sanders, M. Saraceno, B. Sundaram, Phys. Rev. Lett. 63, 2771 (1989).

[16] E.J. Heller, Nature 380, 583 (1996).

[17] S. Sridhar, E.J. Heller, Phys. Rev. A 46, R1728 (1992).

[18] P.B. Wilkinson, T.M. Fromhold, L. Eaves, F.W. Sheard, N. Miura, T. Takamasu, Nature 380, 608 (1996); see also T.M. Fromhold, F.W. Sheard, L. Eaves, P.B. Wilkinson, Nature 387, 864 (1997). 
[19] T.M. Fromhold, L. Eaves, F.W. Sheard, M.L. Leadbeater, T.J. Foster, P.C. Main, Phys. Rev. Lett. 72, 2608 (1994).

[20] T.M. Fromhold, P.B. Wilkinson, F.W.Sheard, L. Eaves, J. Miao, G. Edwards, Phys. Rev. Lett. 75, 1142 (1995):

[21] T.M. Fromhold, A. Fogarty, L. Eaves, F.W. Sheard, M. Henini, T.J. Foster, P.C. Main, G. Hill, Phys. Rev. B 51, 18029 (1995).

[22] D.L. Shepelyansky, A.D. Stone, Phys. Rev. Lett. 74, 2098 (1995).

[23] G. Müller, G.S. Boebinger, H. Mathur, L.N. Pfeiffer, K.W. West, Phys. Rev. Lett. 75, 2875 (1995).

[24] T.M. Fromhold, P.B. Wilkinson, F.W. Sheard, L. Eaves, Phys. Rev. Lett. 78, 2865 (1997).

[25] D. Weiss, K. Richter, A. Menschig, R. Bergmann, H. Schweizer, K. von Klitzing,

G. Weimann, Phys. Rev. Lett. 70, 4118 (1993).

[25] D. Weiss, M.L. Roukes, A. Menschig, P. Grambow, K. von Klitzing, G. Weimann, Phys. Rev. Lett. 66, 2790 (1991).

[26] R. Fleischmann, T. Geisel, R. Ketzmerick, Phys. Rev. Lett. 68, 1367 (1992).

[27] O. Agam, S. Fishman, Phys. Rev. Lett. 73, 806 (1994).

[28] T.M. Antonsen Jr., E. Ott, O. Chen, R.N. Oerter, Phys. Rev. E 51, 111 (1995).

[29] E. Narimanov, A.D. Stone, to be published.

[30] J.V. Nöckel, A.D. Stone, Nature 385, 45 (1997). 